\title{
Análisis Hidrológico de la Cuenca Rocafuerte para Estimar el Potencial Hídrico de la Zona
}

\section{Hydrological Analysis of the Rocafuerte Basin to Estimate the Hydraulic Potential of the Area}

\author{
Jorge Mendoza Cedeño 1 https://orcid.org/0000-0002-1668-9194 \\ Antonio Campos Cedeño 2 https://orcid.org/0000-0003-4266-5169 \\ ${ }^{1}$ Instituto de Posgrado, Universidad Técnica de Manabí, Portoviejo, Ecuador \\ jmendoza9543eutm.edu.ec \\ ${ }^{2}$ Facultad de Ciencias Matemáticas, Físicas y Químicas-UTM, Portoviejo, Ecuador \\ acampos@utm.edu.ec
}

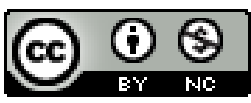

Esta obra está bajo una licencia internacional Creative Commons Atribución-NoComercial 4.0.

\section{Resumen}

El cantón Rocafuerte, fundado en el año1852, es una localidad ubicada en la zona central oeste de la provincia de Manabí. Su principal actividad económica se desarrolla en los ámbitos de la agricultura, ganadería y producción artesanal de dulces, que tienen mucha acogida a nivel nacional e internacional. Uno de los principales problemas de la zona que inciden en su normalidad es el déficit de agua durante los periodos de estiaje, que se presenta, por lo general, entre los meses de junio y noviembre de cada año. Este artículo realiza una valoración del recurso hídrico que se genera en la cuenca donde está asentado el cantón Rocafuerte; esta valoración constituye una información básica para el control, optimización de obras y proyectos tendentes al manejo y uso de dicho recurso. Para el análisis se utilizaron métodos estadísticos y probabilísticos sobre los registros de precipitaciones mensuales que genera el Instituto Nacional de Meteorología e Hidrología del Ecuador (INAMHI). Entre estos métodos se destacan el registro de datos mediante el método de correlación ortogonal; el análisis de consistencia y de variabilidad; la estimación de la precipitación media multianual; la aplicación del método del Servicio de Conservación de Suelos de los E.E.U.U. (SCS) para el cálculo de la escorrentía y la distribución media mensual multianual de la precipitación. Las estaciones meteorológicas que se consideraron dentro del área de estudio fueron 4: Estación Rocafuerte (M165), Portoviejo (M005), Río Chico (M454) y Junín (M462).

Palabras clave: hidrología, precipitaciones mensuales, valoración de la escorrentía, método SCS.
Sumario: 1 Introducción, 2 Materiales y Métodos, 3 Resultados y Discusión, 4 Conclusiones.

Como citar: Mendoza, J., \& Campos, A. (2021). Análisis Hidrológico de la Cuenca Rocafuerte para Estimar el Potencial Hídrico de la Zona. Revista Tecnológica - Espol, 33(1), 10-17.

http://www.rte.espol.edu.ec/index.php/tecnologica/article/view/783 


\begin{abstract}
The Rocafuerte canton, founded in 1852, is a town in the province of Manabí in the central west territory. Its main economic activity is carried out in the fields of agriculture, livestock, and artisanal production of sweets, which are well received national and internationally. One of the main problems in the area that affects normality, is the water deficit during the low water periods, usually between the months of June to November. This work contains an assessment of the water resource that is generated in the basin where the Rocafuerte canton is located and that constitutes basic information for the control, optimization of works and projects aimed at the management and use of that resource. For the analysis, statistical and probabilistic methods were used in the management of the monthly rainfall records provided by the National Institute of Meteorology and Hydrology of Ecuador (INAMHI, acronyms in Spanish), among which the following stand out: data filling using the orthogonal correlation method, analysis of consistency and variability, estimation of multi-year average precipitation, application of the US Soil Conservation Service (SCS) method to calculate runoff and the multi-year monthly average distribution of precipitation. The meteorological stations in this study were 4, namely: Rocafuerte station (M165), Portoviejo (M005), Rio Chico (M454), and Junín (M462).
\end{abstract}

Keywords: Hydrology, monthly precipitation, runoff assessment, SCS method.

\title{
1. Introducción
}

Los estudios más recientes relacionados con la determinación del potencial hídrico de la Demarcación Hidrográfica de Manabí, que incluye a la zona de Rocafuerte, fueron realizados en 1990 por instituciones de desarrollo, como el Centro de Rehabilitación de Manabí (CRM), la Organización de Estados Americanos (OEA) y la Corporación Ultramar de Japón (JICA). El resultado de estos estudios se publicó en el Plan Integral de Desarrollo de los Recursos Hídricos de Manabí (PHIMA) (Centro de Rehabilitación de Manabí et al., 1989), que se ha constituido en la principal guía para quienes se dedican a realizar investigaciones y estudios sobre la optimización y el aprovechamiento de los recursos hídricos de Manabí.

Han transcurrido 30 años desde esa fecha sin que se haya actualizado dicha información. Esta investigación no solo constituye una actualización, sino que profundiza en aspectos relevantes encaminados a determinar el potencial hídrico de la zona, información preponderante dentro del marco de la planificación y el uso del recurso hídrico.

Territorialmente, la República del Ecuador se encuentra dividida en 24 provincias, de las cuales 7 pertenecen a la región Costa y, entre ellas, se cuenta la denominada provincia de Manabí, que tiene una extensión aproximada de 19 mil km², lo cual representa un $6.64 \%$ de todo el territorio nacional. A su vez, la provincia de Manabí está formada por 22 cantones.

De todas las provincias del país, la más deficitaria en lo que a recursos hídricos se refiere es la provincia de Manabí, debido a que la divisoria de la cuenca interviene como una barrera que evita el acceso a la provincia de aguas superficiales, provenientes del deshielo de la Cordillera de los Andes. Esta situación ha conllevado a la identificación e implementación de proyectos tendentes al almacenamiento del agua producida en la estación de lluvias, para su posterior uso en épocas de sequias. Uno de los proyectos (Horton, 1945) que se encuentra en ejecución desde los años 70 es el Proyecto Integrado Chone-Portoviejo, que atiende a las necesidades hídricas, tanto para riego como para consumo humano, de la zona central de la provincia de Manabí.

Esta investigación, que tiene por objeto central valorar la precipitación y escorrentía de la cuenca en la que se encuentra asentada la parroquia Rocafuerte, constituye un aporte a la solución del problema del déficit de agua existente en la zona. Los afluentes tributarios a esta cuenca, a la que la Secretaría del Agua ha codificado como la unidad hidrológica "15142", tiene como afluentes a los esteros El Guanábano, La Papaya y Ojo de Agua, que tienen su desembocadura en el río Bachillero, el que, a su vez, confluye en el río Portoviejo, próximo al océano Pacífico. 
Este trabajo se ofrece como insumo a las instituciones encargadas del desarrollo nacional encargadas del desarrollo nacional, el control y la optimización de los recursos hídricos existentes.

\section{Materiales y Métodos}

Los aspectos metodológicos más relevantes en el presente trabajo contemplan: 1) Selección de las estaciones meteorológicas del Ecuador (INAMHI, 2015; Secretaría de Gestión de Riesgos, 2014); 2) Recolección de registros de precipitaciones mensuales; 3) Análisis de consistencia de datos (Rusticucci y Barrucand, 2001); 4) Registro de datos faltantes en las series (Romero y Casimiro, 2015), 5) Análisis de variabilidad (Valinova, 2016); 6) Estimación de la precipitación media multianual (Campos et al., 2015); 7) Valoración del volumen de agua por precipitación; 8) Estimación de la precipitación de la cuenca (Chow, 1988; Verdú et al., 2006); 9) Estimación de la escorrentía en la cuenca (ARES et al., 2012) y 10) Distribución mensual de la precipitación media.

La información básica recabada para el análisis y desarrollo de la investigación está compuesta de los registros pluviométricos de precipitaciones mensuales tomados de las estaciones meteorológicas Rocafuerte (M165), Portoviejo (M005), Río Chico (M454), Junín (M462). Dicha información fue proporcionada por el Instituto Nacional de Meteorología e Hidrología (INAMHI). El total de registros por estación correspondiente a la precipitación mensual para el periodo de análisis 1963-2017 fue de 660 lo que sumó un total de 2640 registros entre las 4 estaciones.

Las estaciones meteorológicas del estudio se encuentran localizadas en los límites de la Unidad Hidrológica de la Demarcación Hidrográfica de Manabí, identificada con el código Pfafstetter "15142".

En la Figura 1 se presenta la ubicación geográfica de las mencionadas estaciones, ubicadas entre las coordenadas UTM WGS84 y el cuadrante 17S.

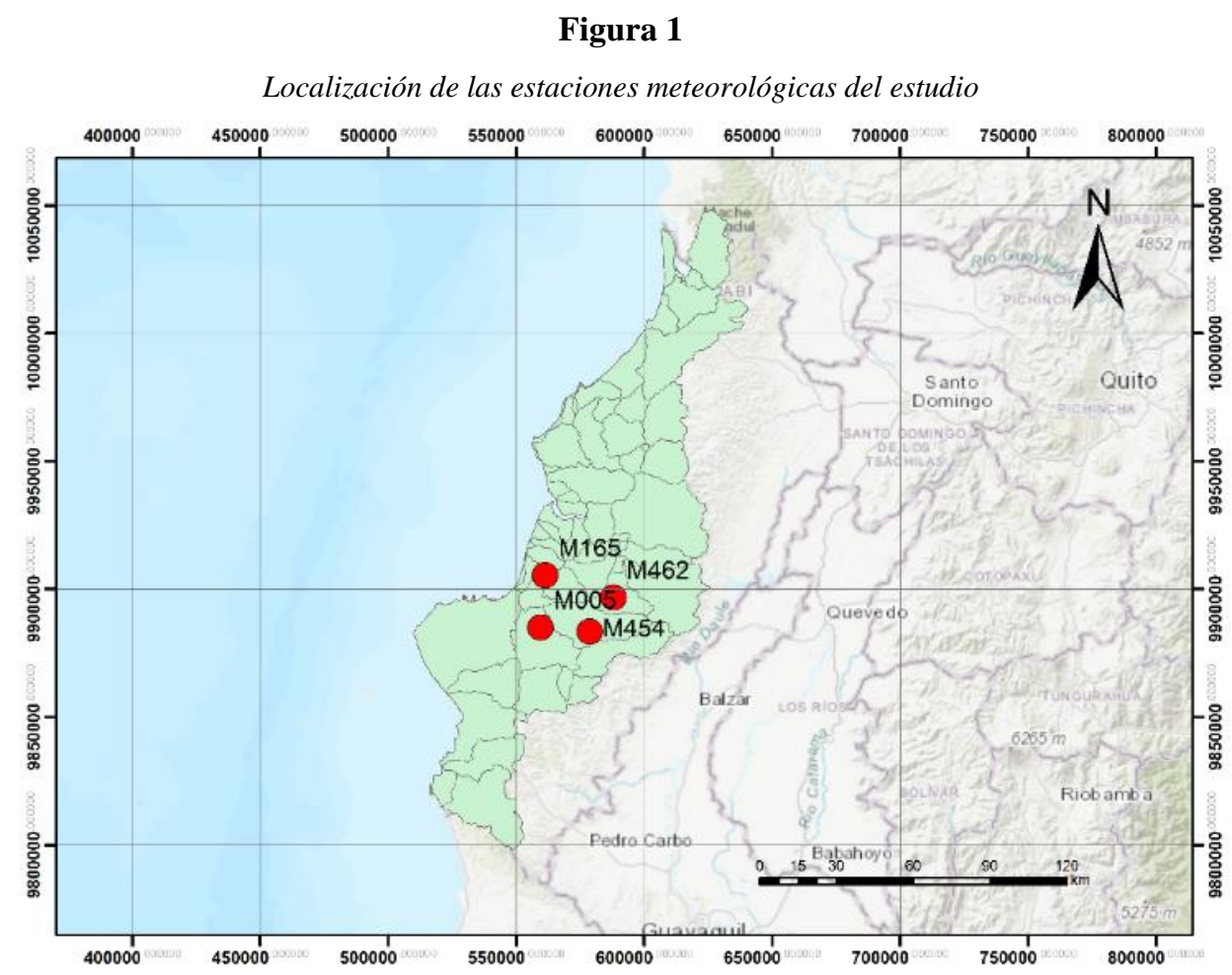

Previo al desarrollo de la investigación, se procedió a escoger los registros y a analizar su consistencia y variabilidad. Para la selección de las estaciones se utilizaron los polígonos de Thiessen (Campos A.F. y Sinichenko E.K., 2017). Para el análisis de consistencia se elaboraron las curvas de masa. El análisis de variabilidad se sustentó con el cálculo de coeficiente de variación a partir de la fórmula: 
Donde:

$$
C_{v}=\frac{\sigma}{\bar{x}}
$$

- $C_{v}-$ Coeficiente de variación,

- $\underline{\sigma}$ - Desviación estándar,

- $\mathrm{x}$ - Media aritmética.

Se ha establecido que, si el coeficiente de variación es inferior a 0.30 , se considera a la serie uniforme (Valinova, 2016).

La precipitación media multianual de la cuenca se la determina con la siguiente expresión:

Donde:

$$
P_{m m a}=\frac{\sum_{i=1}^{n} P_{i} A_{i}}{\sum_{i=1}^{n} A_{i}}
$$

- P_mma - Precipitación media multianual de la cuenca, [mm]

- Pi - Precipitación media multianual de cada microcuenca, [mm]

- $\mathrm{Ai}$ - Área de cada microcuenca, $[\mathrm{km} 2]$

- $\mathrm{n}$ - Cantidad de microcuencas

El volumen de agua, resultado de la precipitación total, se lo obtiene de la precipitación media multianual de la cuenca por su respectiva área.

La escorrentía se la estimó mediante el modelo hidrológico precipitación-escorrentía propuesto por el Servicio de Conservación de Suelo de los EE. UU. (SCS) (Department of Agriculture United States, 2015), llamado hoy Servicio de Conservación de Recursos Naturales (Natural Resources Conservation Service-NRCS). Este método requiere la aplicación de las siguientes formulas:

Donde:

$$
Q e=\frac{(P-0.2 S)^{2}}{P+0.8 S}
$$

- Qe - Escorrentía efectiva, [mm]

- P - Precipitación total, [mm

- S - Diferencia potencial máxima entre P y Q, [mm]

Donde:

$$
S=25400 / C N
$$

- $\mathrm{CN}$ - Numero de curva, en función del tipo de suelo, usos y cobertura vegetal (Cedeno et al., 2019).

Por el hecho de que la zona de estudio se encuentra ubicada en la zona ecuatorial, el clima con el que se cuenta es de carácter tropical, lo que determina que fundamentalmente existan en el año dos periodos climáticos, denominados comúnmente como lluvioso y seco. El periodo lluvioso, por lo general, se inicia en diciembre y termina en junio, mientras que el seco predomina en los meses restantes (julio-noviembre). Esta situación contribuye a la existencia de una irregular y desproporcionada distribución de las precipitaciones a lo largo del año. Para poder tener un panorama del comportamiento medio de las precipitaciones mensuales multianuales se determinaron las medias aritméticas de cada mes para la serie de datos ponderada.

\section{Resultados y Discusión}

Las estaciones meteorológicas escogidas para el análisis hidrológico son aquellas que corresponden a los polígonos de Thiessen, en los que se encuentra enmarcada el área de estudio. Dichas estaciones son Rocafuerte (M165), Portoviejo (M005), Río Chico en Alajuela (M454) y Junín (M462). 
El total del área de la cuenca estudiada asciende a $146.74 \mathrm{~km}^{2}$. Dicha área está constituida por 4 sectores, de los cuales la mayoría pertenece a la estación Rocafuerte, con una superficie de $119.12 \mathrm{~km}^{2}$.

Para las estaciones seleccionadas se obtuvieron del INAMHI los registros mensuales de precipitación para el periodo 1963-2017. Para cada estación se contó con, aproximadamente, 600 registros.

Previo a registro de datos faltantes, que se lo hizo mediante el método de correlación ortogonal, se llevó a cabo el respectivo análisis de consistencia, mediante el cual se llegó a determinar la existencia de la correlación de los registros escogidos. Esta tarea se la ejecutó mediante la elaboración de las curvas de masa.

Para el análisis de variabilidad, los registros de cada estación fueron sometidos al cálculo del coeficiente de variación, obteniéndose los valores de: $0.62,0.65,0.49$ y 0.54 para las estaciones de Portoviejo, Rocafuerte, Río Chico y Junín, respectivamente. El hecho de haber obtenido valores de coeficiente de variación superiores a 0.30 indica una distribución no uniforme de los registros.

Considerando que el área total de la cuenca es igual a $146.74 \mathrm{~km}^{2}$ y que la precipitación media multianual ha sido estimada en $556,55 \mathrm{~mm}$, el volumen medio multianual producido por las precipitaciones ha sido valorado en $81.67 \mathrm{hm}^{3}$. La precipitación media multianual de la cuenca alcanzó $556.55 \mathrm{~mm}$. En la Tabla 1 se presentan los resultados parciales por microcuencas.

Tabla 1

Precipitación media multianual de la cuenca

\begin{tabular}{|ccccc|}
\hline Estación & Código & $\begin{array}{c}\text { Área } \\
{\left[\mathbf{k m}^{2}\right]}\end{array}$ & $\begin{array}{c}\text { Precipitación } \\
{[\mathbf{m m}]}\end{array}$ & $\boldsymbol{P}^{*} \boldsymbol{A}$ \\
\hline Portoviejo-UTM & M005 & 0.13 & 527.79 & 70.14 \\
Rocafuerte & M165 & 119.12 & 454.58 & 54149.14 \\
Rio Chico & M454 & 7.72 & 862.76 & 6660.48 \\
Junín & M462 & 19.77 & 1051.59 & 20789.88 \\
\hline$\Sigma$ & & 146.74 & & 81669.64 \\
\hline & & $\mathbf{P m m a}=$ & $\mathbf{5 5 6 . 5 5}$ & $\mathbf{m m}$ \\
\hline
\end{tabular}

Fuente: Elaboración propia

Sobre la base de los mapas de suelo y cobertura vegetal del Ecuador, desarrollados por el Ministerio de Agricultura y Ganadería (MAG), el Instituto Interamericano de Cooperación para la Agricultura (IICA) y el Centro de Levantamientos Integrados de Recursos Naturales por Sensores Remotos (CLIRSEN), se obtuvo el mapa de los números de curvas para el territorio ecuatoriano, insumo fundamental para la estimación de la escorrentía, de conformidad con la metodología de U.S. Soil Conservation Service (Campos A.F. \& Sinichenko E.K., 2017). La capa por escorrentía anual determinada por el método SCS se la ha estimado en $2631.09 \mathrm{~mm}$. Por otro lado, el valor del número de curva medio, de conformidad con el mapa elaborado para la zona, resultó ser igual a 81 , con lo cual se obtuvo que la escorrentía anual de la cuenca está por el orden de $72.11 \mathrm{hm}^{3}$, lo cual constituye 87.8 $\%$ del total de precipitaciones.

En la Tabla 2 se condensan los resultados parciales y totales de la escorrentía de la cuenca del rio Rocafuerte.

Para cada una de las estaciones se determinó el porcentaje multianual respecto al total medio, y, luego, con la ayuda de las respectivas áreas, se llegó a establecer valores ponderados para la cuenca en general. La Tabla 3 contiene los porcentajes parciales calculados.

De la Tabla 3, en función de los valores ponderados, se desprende que la mayor concentración de precipitación se da en el mes de febrero, con un porcentaje equivalente al $28.18 \%$, mientras que el valor más deficitario del recurso hídrico se dio en el mes de octubre, con un valor de $0.53 \%$. 
Tabla 2

Escorrentía de la cuenca

\begin{tabular}{|cccccccc|}
\hline Estación & Código & $\begin{array}{c}\text { Prep. } \\
{[\mathbf{m m}]}\end{array}$ & $\begin{array}{c}\text { Área } \\
{\left[\mathbf{k m}^{2}\right]}\end{array}$ & $\boldsymbol{C N}$ & $\mathbf{S}$ & $\begin{array}{c}\text { Qe } \\
{[\mathbf{m m}]}\end{array}$ & $\begin{array}{c}\text { Vesc } \\
{\left[\mathbf{h m}^{2}\right]}\end{array}$ \\
\hline Portoviejo & M005 & 527.79 & 0.13 & 81.00 & 59.58 & 462.46 & 0.06 \\
Rocafuerte & M165 & 454.58 & 119.12 & 81.00 & 59.58 & 390.15 & 46.47 \\
Rio Chico & M454 & 862.76 & 7.72 & 81.00 & 59.58 & 795.16 & 6.14 \\
Junín & M462 & 1051.59 & 19.77 & 81.00 & 59.58 & 983.32 & 19.44 \\
\hline$\sum$ & & & & & & 2631.09 & 72.11 \\
\hline
\end{tabular}

Fuente: Elaboración propia

Del análisis se desprende también que existe una gran desproporción en la distribución de la precipitación por periodos climáticos, ya que en la estación lluviosa (diciembre a junio) se concentra el $93.52 \%$ del total anual, mientras que en la estación seca (julio a noviembre) su porcentaje llega al 6.48 $\%$.

Tabla 3

Distribución porcentual mensual de la precipitación por microcuencas

\begin{tabular}{|c|c|c|c|c|c|c|c|c|c|c|c|c|c|}
\hline CÓDIGO & $\begin{array}{l}\text { AREA } \\
{\left[\mathbf{k m}^{2}\right]}\end{array}$ & ENE & \multicolumn{10}{|c|}{ [Porcentaje respecto al total] } & DIC \\
\hline M005 & 0.13 & 17.95 & 25.31 & 25.07 & 13.68 & 6.04 & 3.25 & 1.39 & 0.52 & 0.68 & 0.58 & 1.17 & 4.36 \\
\hline M165 & 119.1 & 17.79 & 28.69 & 23.59 & 13.38 & 6.15 & 2.29 & 1.26 & 0.74 & 0.61 & 0.48 & 1.05 & 3.98 \\
\hline M454 & 7.72 & 16.65 & 25.14 & 22.50 & 16.32 & 7.11 & 3.16 & 1.25 & 0.82 & 0.85 & 1.01 & 1.17 & 4.03 \\
\hline M462 & 19.77 & 17.65 & 26.32 & 23.79 & 15.98 & 5.93 & 2.00 & 1.43 & 0.69 & 0.38 & 0.65 & 1.01 & 4.16 \\
\hline Valores Po & derados & 17.71 & 28.18 & 23.57 & 13.89 & 6.17 & 2.29 & 1.28 & 0.74 & 0.59 & 0.53 & 1.05 & 4.00 \\
\hline
\end{tabular}

Fuente: Elaboración propia

En la Tabla 4 se indican los valores generales obtenidos en el análisis de la distribución media multianual mensual de las precipitaciones.

Tabla 4

Distribución mensual de la precipitación media multianual

\begin{tabular}{|c|c|c|c|c|c|}
\hline \multirow{2}{*}{ Mes } & \multicolumn{2}{|c|}{ Precipitación } & \multirow{2}{*}{ Estación } & \multicolumn{2}{|c|}{ Precipitación total } \\
\hline & {$[\%]$} & {$[\mathrm{mm}]$} & & {$[\mathrm{mm}]$} & {$[\%]$} \\
\hline Diciembre & 4.00 & 22.28 & \multirow{6}{*}{ 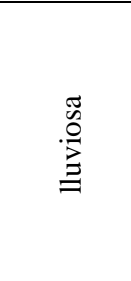 } & \multirow{6}{*}{520.46} & 93.52 \\
\hline Enero & 17.71 & 98.58 & & & \\
\hline Febrero & 28.18 & 156.82 & & & \\
\hline Marzo & 23.57 & 131.15 & & & \\
\hline Abril & 13.89 & 77.30 & & & \\
\hline Mayo & 6.17 & 34.32 & & & \\
\hline Junio & 2.29 & 12.77 & \multirow{6}{*}{$\underset{\mathscr{W}}{\overparen{W}}$} & \multirow{6}{*}{36.09} & 6.48 \\
\hline Julio & 1.28 & 7.12 & & & \\
\hline Agosto & 0.74 & 4.11 & & & \\
\hline Septiembre & 0.59 & 3.30 & & & \\
\hline Octubre & 0.53 & 2.95 & & & \\
\hline Noviembre & 1.05 & 5.84 & & & \\
\hline$\sum$ & 100.00 & 556.55 & & 556.55 & 100.00 \\
\hline
\end{tabular}

Fuente: Elaboración propia 


\section{Conclusiones}

La escorrentía superficial que se produce en la cuenca de la unidad hidrológica "15142" se ha estimado que asciende a los $72.11 \mathrm{hm}^{3}$ anuales; este cálculo se obtuvo restándole a la precipitación total el valor correspondiente a la filtración de conformidad con el tipo de suelo, cobertura vegetal y uso del territorio de la zona.

La precipitación total media multianual, obtenida del análisis de los registros pluviométricos mensuales para el periodo 1963-2017, fue estimada en $81.67 \mathrm{hm}^{3}$.

Se llegó a establecer en términos generales que existe una gran variabilidad en la distribución mensual de las precipitaciones. En el mes de febrero alcanza su valor máximo, $28 \%$, y, en octubre, su valor mínimo, $0.53 \%$.

Tanto para los periodos lluvioso y seco se determinó la tasa de acumulación de la precipitación, llegando a la conclusión de que el $93.52 \%$ se acumula en la temporada lluviosa (diciembre-junio), mientras que, para el periodo de estiaje que se presenta entre los meses de julio a noviembre, el aporte baja considerable a niveles estimados en $6.48 \%$.

La población del cantón Rocafuerte, estimada en el último censo poblacional del año 2010, llevado a cabo por el Instituto Nacional de Estadística y Censos del Ecuador (INEC, 2020), alcanzó la suma de 33736 habitantes, con una tasa de crecimiento equivalente al $1.1 \%$. Con la ayuda de estos datos se estimó una población futura de 52256 habitantes en el año 2050. Por otro lado, teniendo en consideración la incorporación al riego de 1500 ha, según las proyecciones que se ha realizado a partir de los datos oficiales, y estableciendo una norma media de riego equivalente al $1.77 \mathrm{l} / \mathrm{s} / \mathrm{ha}$, de manera general, se llegó a identificar los volúmenes anuales de agua que se requerirían para cubrir estas necesidades vitales de consumo humano y riego. La cifra estimada necesarias para esta última actividad asciende a la cantidad de $27.53 \mathrm{hm}^{3}$.

Comparando la oferta hídrica obtenida $\left(72.11 \mathrm{hm}^{3}\right)$ con la demanda $\left(27.53 \mathrm{hm}^{3}\right)$ se concluye que los recursos hídricos anuales que se producen en la cuenca "15142" resultan ser suficientes para el desarrollo productivo de la zona, para lo cual, por supuesto, se requiere proyectar y construir las obras requeridas de regulación y almacenamiento del agua durante el periodo lluvioso para, luego, emplearlas durante la estación de estiaje.

\section{Referencias}

ARES, G., Chagas, C., \& Varni, M. J. C. d. s. (2012). Predicción de la Escorrentía y Estimación de la Humedad Antecedente en una Cuenca Aforada. 30(2).

Campos A.F., \& Sinichenko E.K. (2017). Características de Sistemas Fluviales Pequeños y Recursos Hídricos de la Demarcación Hidrográfica de Manabí, Perspectivas de Desarrollo. Universidad de la Amistad de los Pueblos de Rusia.

Campos, A. F., Sinichenko, E. K., \& Gritsuk, I. I. (2015). Modelo matemático para la determinación de las precipitaciones medias anuales con la ayuda de rasters y otros elementos GIS. VESTNIK № 3 - Peoples' Friendship University of Russia, 9.

Cedeno, A. F. C., Guillen, P. A. S., Ramos, J. L. M., Sinichenko, E. K., \& Gritsuk, I. I. (2019). Estimation of the runoff of the hills of the city of PortoviejoEcuador to assess the degree of flooding in the region. IOP Conference Series: Materials Science and Engineering,

Centro de Rehabilitacion de Manabí, INERHI, CONADE, \& OEA. (1989). Plan Integral de Desarrollo de los Recursos Hídricos de la Provincia de Manabí (PHIMA) (Vol. Resumen Ejecutivo). Centro de Rehabilitación de Manabí.

Chow, V. T. (1988). Applied Hydrology. University of Illinois. 
Ecuador (INAMHI), I. N. d. M. e. H. d. (2015). Determinación de ecuaciones para el cálculo de intensidades máximas de precipitación. INAMHI.

Horton, R. E. (1945). Erosional development of streams and their drainage basins; hydrophysical approach to quantitative morphology. Geological Society of America.

Romero, A. E. L., \& Casimiro, W. S. L. J. R. T.-E. (2015). Evaluación de métodos hidrológicos para la completación de datos faltantes de precipitación en estaciones de la cuenta Jetepeque, Perú. 28(3).

Rusticucci, M., \& Barrucand, M. J. M. (2001). Climatología de temperaturas extremas en la Argentina. Consistencia de datos. Relación entre la temperatura media estacional y la ocurrencia de días extremos. 26, 69-84.

Secretaría de Gestión de Riesgos. (2014). Anuarios Meteorológicos. Retrieved 19.11.2014 from http://186.42.174.231/index.php/clima/anuarios-meteorologicos

Valinova, V. S. (2016). Coeficiente de variación y coeficiente de determinación. Retrieved 15.05.2016 from http://univer-nn.ru/statistika/koefficient-variacii/

Verdú, J. M., Batalla, R. J., \& Martínez Casasnovas, J. A. J. I. d. a. (2006). Estudio hidrológico de la cuenca del río Isábena (Cuenca del Ebro). I: Variabilidad de la precipitación. 13(4), 321-330. 